\title{
CORRECTION
}

Check for updates

\section{Correction: C3a and C5a facilitates the metastasis of myeloma cells by activating Nrf2}

Jie Xiong (D), Xingyi Kuang, Tingting Lu, Kunlin Yu, Xu Liu (D), Zhaoyuan Zhang, Weili Wang (D), Lu Zhao, Qin Fang, Depei Wu and Jishi Wang (iD

(c) The Author(s), under exclusive licence to Springer Nature America, Inc. 2021

Cancer Gene Therapy (2021) 28:1227; https://doi.org/10.1038/s41417-021-00361-1

Correction to: Cancer Gene Ther

https://doi.org/10.1038/s41417-020-00217-0

In the published article, errors have been identified in the sections listed below.

In the Figure 4(i),
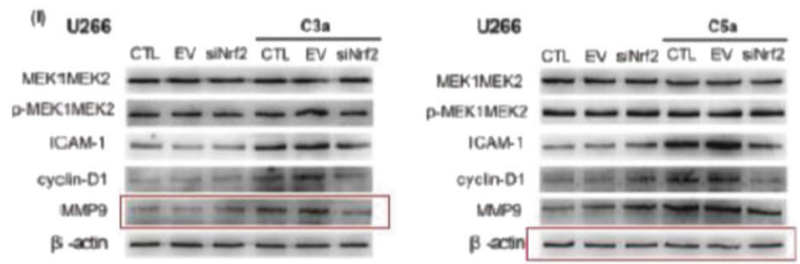

In the Figure 4(j),

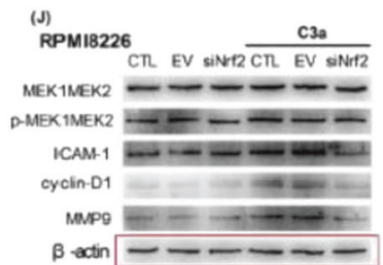

The bands in Figure 4(i) and 4(j) should be:

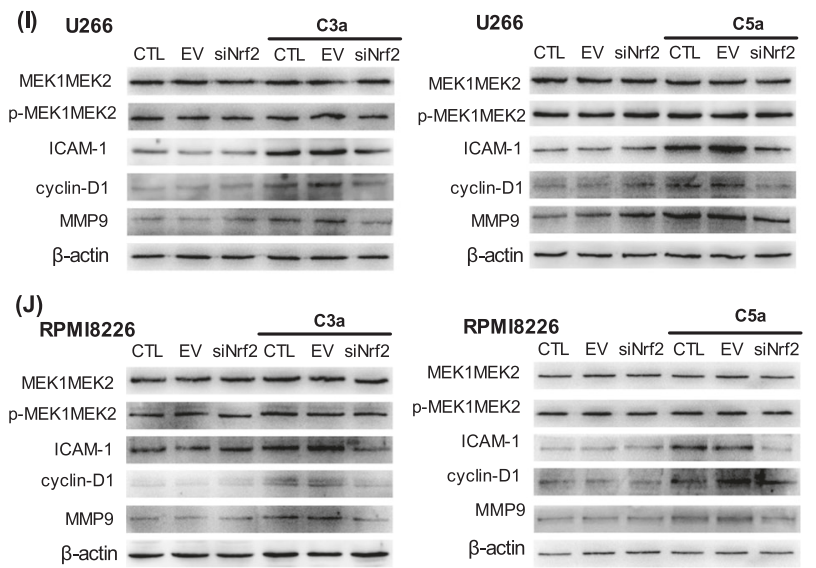

The original article has been corrected. 